\title{
Unified Description of Coupled Resonators and Coupled Transmission Lines
}

\author{
A. ABramowicz* \\ Institute of Electronic Systems, Warsaw University of Technology \\ Nowowiejska 15/19, 00-665 Warsaw, Poland
}

\begin{abstract}
Coupled resonators and coupled transmission lines are described with the same coupling curve. A ratio between resonant frequencies, squared, of coupled resonators and ratio between impedances of coupled transmission lines reveal the same type of dependence on coupling coefficient. This can be used to select, adjust or create the models of coupled structures. As an example the new model of coupled transmission lines has been developed. Its application in LC element directional coupler is presented and discussed.
\end{abstract}

PACS: 84.40.Dc

\section{Introduction}

Microwave resonators and microwave transmission lines can be coupled to create frequency selective structures used in microwave devices. Main applications of coupled transmission lines and resonators are filters but also couplers, transformers and many other devices. In the past coupled structures were described by circuit models $[1,2]$. Such models were very helpful in design of microwave circuits and still are used. As an example of important circuit theory that is still used and even under continuous development one can consider the multiple coupled resonator filter design method. In the method the coupling matrix of the filter is designed based on the use of ideal inverters approximating couplings between resonators [3]. Now microwave structures are mostly analyzed and designed in electromagnetic simulators [4-6] but anyway circuit models can help in speeding up the analysis.

Microwave filters can be built of different resonators: cavities, dielectric resonators, planar resonators, comb-line and interdigital resonators, LC resonant circuits etc. Some of the filter structures apply coupled transmission lines serving as coupled resonators e.g. comb-line and interdigital resonators, parallel-coupled resonators. This motivated the author to search for similarities and differences in theory describing coupling effects between coupled resonators and coupled transmission lines. Obtained results seem to be very interesting because as it is shown below the unified description of the electromagnetically coupled structures can be found. Such a unified description can be used to improve the modelling of coupled structures and improve the design process of de-

\footnotetext{
* e-mail: aabr@ise.pw.edu.pl
}

vices applying coupled resonators and/or coupled transmission lines. It has been found that there is a universal curve of coupling coefficient applicable to both coupled resonators and coupled transmission lines. This implies that the same formulae can be used to describe resonant frequencies of coupled resonators and impedances of coupled transmission lines. Taking advantage of the coupled resonators theory new models of coupled transmission lines are introduced and discussed. Their circuit equivalents are presented and analyzed. As a result novel structures of LC directional couplers are obtained.

\section{Resonant frequencies of coupled resonators}

Two coupled resonators or resonant circuits have two resonant frequencies [7-9]. These resonant frequencies (called also eigenfrequencies) can be accurately computed or measured. Let us consider two identical resonators, which are coupled and isolated from any other structures. When the coupling is small, the resonant frequencies are close to the resonant frequency of uncoupled resonators. When the strength of coupling increases, the difference between two resonant frequencies of coupled resonators increases as well. The strength of coupling is described with the coupling coefficient that can be computed from the following formula [7-9]:

$$
k=\frac{\left|f_{2}^{2}-f_{1}^{2}\right|}{f_{1}^{2}+f_{2}^{2}},
$$

where $f_{1}$ and $f_{2}$ are resonant frequencies of coupled resonators. The resonant frequencies can be precisely found from electromagnetic simulations. In fact the resonant frequencies can be computed analyzing a half of the coupled resonator structure with perfect electric or perfect magnetic wall introduced in the symmetry plane. Depending on the type of coupling the eigenfrequency $f_{1}$ 
can be lower or higher than $f_{2}$. On the base of circuit models it is assumed that magnetic type of coupling results in $f_{2}>f_{1}$ and that electric type of coupling produces reversed relation between eigenfrequencies. For the purpose of this paper let us assume that $f_{2}>f_{1}$. Then the formula (1) can be written without absolute value sign. The reason for such an assumption is explained in Sect. 3 .

In MIKON 2010 paper [10], three examples of typical microwave structures: coupled rectangular waveguide resonators, coupled comb-line resonators and coupled interdigital resonators have been shown. They have been analyzed by means of QuickWave; a general-purpose electromagnetic simulator based on FDTD method [11]. Resonant frequencies of presented structures and calculated coupling coefficients exhibiting different behaviour of the resonant frequencies and different frequency range have been used to create Fig. 1 where the coupling coefficient is drawn versus the ratio between resonant frequencies squared. Not surprisingly the characteristic of coupling coefficient is independent of the type of coupled resonators. After a simple transformation Eq. (1) is as follows:

$$
k=\frac{1-\frac{f_{1}^{2}}{f_{2}^{2}}}{\frac{f_{1}^{2}}{f_{2}^{2}}+1}=g\left(\frac{f_{1}^{2}}{f_{2}^{2}}\right),
$$

where $g$ means the function, which is the same for any coupled resonators. Thus one can state that coupling coefficient depends on the ratio between resonant frequencies. It seems obvious that for any resonator structure the coupling coefficient depends on the ratio between resonant frequencies squared in the same way as shown in Fig. 1.

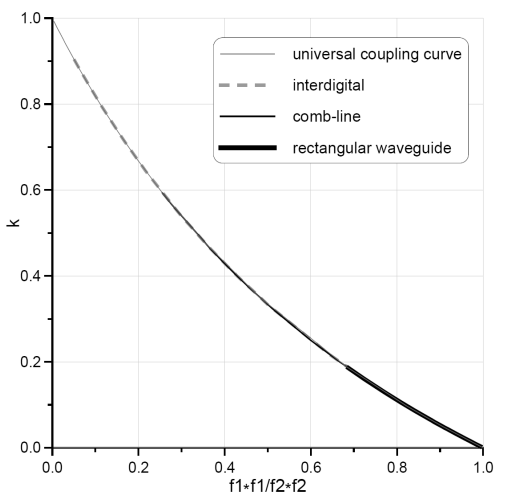

Fig. 1. Coupling coefficient versus ratio between resonant frequencies squared.

From Eq. (2) it is not difficult to find that the ratio between resonant frequencies squared depends on coupling coefficient as

$$
\frac{f_{1}^{2}}{f_{2}^{2}}=g^{-1}(k)=\frac{1-k}{1+k} .
$$

\section{Impedances of coupled transmission lines}

In a case of coupled symmetric transmission lines electromagnetic coupling produces two modes of propagation. The modes are named "odd" and "even" $[12,13]$. Each mode has its own characteristic impedance denoted $Z_{0 \mathrm{o}}$ for odd mode and $Z_{0 \mathrm{e}}$ for the even mode. When coupling is small the impedances are close to the impedance of uncoupled lines. When the strength of coupling increases, the difference between impedances becomes bigger. The coupling coefficient of coupled transmission lines can be calculated from their impedances [12]:

$$
k=\frac{Z_{0 \mathrm{e}}-Z_{0 \mathrm{o}}}{Z_{0 \mathrm{o}}+Z_{0 \mathrm{o}}} .
$$

It should be noted that the odd mode impedance $Z_{0 \mathrm{o}}$ is always lower than the impedance of the even mode $Z_{0 \mathrm{e}}$. This is the reason for assuming the inequality $f_{2}>$ $f_{1}$ in the previous paragraph. In MIKON paper [10] examples of the coupled transmission lines have been presented. In Fig. 2 one more example is shown.

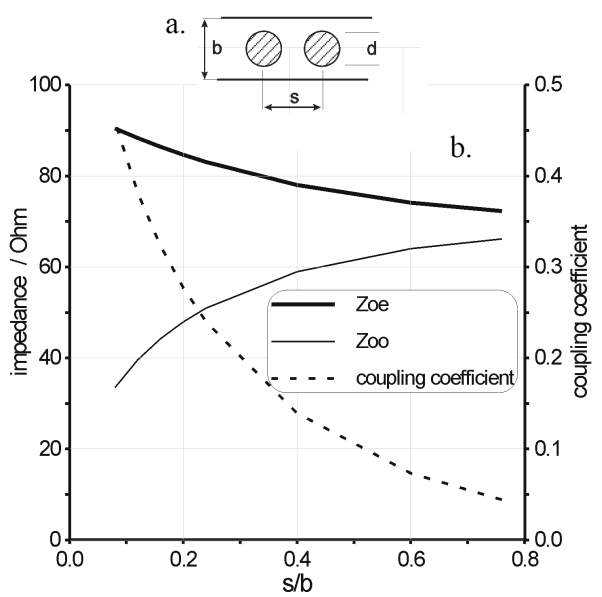

Fig. 2. (a) Coupled slab lines. (b) Corresponding impedances and coupling coefficient.

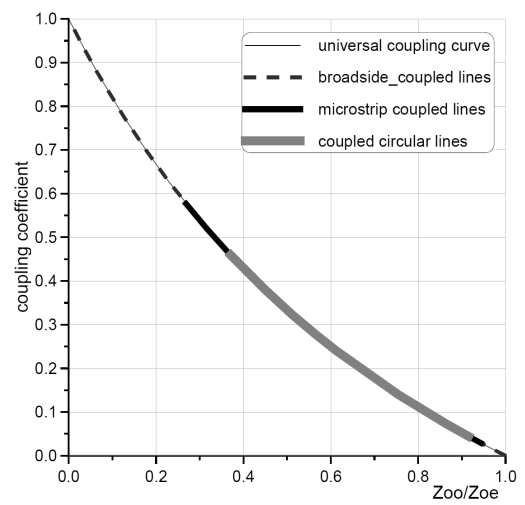

Fig. 3. Coupling coefficient versus ratio between impedances $Z_{0 \mathrm{o}}$ and $Z_{0 \mathrm{e}}$. 
Impedances and coupling coefficients of coupled slab lines shown structures are presented. The impedances have been computed obtained from conformal transformation method [14]. Computed impedances from [10] and Fig. 2 have been used to create a plot shown in Fig. 3 where a coupling coefficient versus ratio between the impedances $Z_{0 \text { o }}$ and $Z_{0 \text { e }}$ has been drawn. The same curve as in a case of coupled resonators has been obtained which is not surprising taking into account the similarity of formulae (4) and (1). Let us transform Eq. (4):

$$
k=\frac{1-\frac{Z_{1}}{Z_{2}}}{\frac{Z_{1}}{Z_{2}}+1}=g\left(\frac{Z_{1}}{Z_{2}}\right),
$$

where $g$ is the same function as in Eq. (2) and the same one for any coupled transmission lines. Finally it is not difficult to find that the ratio between impedances depends on coupling coefficient as

$$
\frac{Z_{0 \mathrm{o}}}{Z_{0 \mathrm{e}}}=g^{-1}(k)=\frac{1-k}{1+k} .
$$

Presented coupled transmission lines are typical examples. All uniform coupled transmission lines revealed the same behaviour shown in Fig. 3. Thus one can state that the shape of the curves from Fig. 1 and Fig. 3 is the universal feature of both resonator and transmission line coupled structures.

\section{Implications}

The universal curve describing both coupled resonators and coupled transmission lines can be used to create and verify circuit models of coupled structures. The dependence of the resonant frequencies squared and impedances on coupling coefficient in any model should comply with the formula (3) and (6). The universal curve can be also used to improve the accuracy of computed resonant frequencies and impedances.

The circuit models of coupled microwave resonators are well known. Over 10 circuit models can be found in the literature $[7-9,15]$. The eigenfrequency method for computation of coupling coefficient between microwave resonators is successfully used in the microwave filter design methods. The method is based on the circuit models of coupled resonators. The circuit models can be divided into simple i.e. using an inverter with only one type of immitance and combined i.e. using the inverter with two different immitances $[7-9,15]$. As an example let us consider two resonant circuits coupled through inverter with series capacitance between resonators, as shown in Fig. 4. The circuit from Fig. 4 has two resonant frequencies

$$
\begin{aligned}
& f_{1}^{2}=f_{0}^{2} \frac{1}{1+k}, \\
& f_{2}^{2}=f_{0}^{2} \frac{1}{1-k},
\end{aligned}
$$

where coupling coefficient $k=C_{\mathrm{s}} / C$, resonant frequency of uncoupled resonators $f_{0}=1 /(2 \pi \sqrt{L C})$. The ratio between resonant frequencies squared is described by Eq. (3). Thus the model complies with the universal coupling curve.

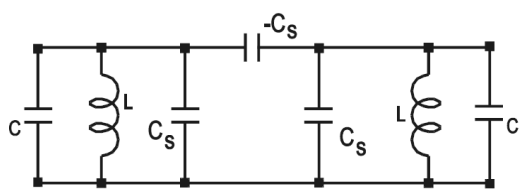

Fig. 4. Parallel resonant circuits coupled through admittance inverter with capacitances.

Coupled transmission lines are described with the only one circuit model $[12,13,16]$. In this model introduced by Oliver [13] the coupling between line of unit inductance $L$ and unit capacitance $C$ is provided through admittance inverter with series negative capacitance $C_{\mathrm{s}}$ and impedance inverter with parallel inductance $L_{\mathrm{p}}$. From the Oliver model the following impedances of the odd and even mode can be found [17]:

$$
\begin{aligned}
& Z_{0 \mathrm{o}}=Z \sqrt{\frac{1-k}{1+k}}, \\
& Z_{0 \mathrm{e}}=Z \sqrt{\frac{1+k}{1-k}},
\end{aligned}
$$

where coupling coefficient $k=L_{\mathrm{p}} / L$ and $k=C_{\mathrm{s}} / C$, impedance $Z$ is the impedance of a single, uncoupled line $Z=\sqrt{L / C}$. Again, computing the ratio between modal impedances Eq. (6) is obtained. The model complies with the universal coupling curve. Despite the unphysical properties of the Oliver model described in [17] it has one more unpleasant feature i.e. negative series capacitance that is introduced between lines. It is impossible to create a real LC structure due to negative series capacitance.

\section{New model of coupled transmission lines}

It would be interesting to create a new model of coupled transmission lines that has proper physical properties and also can be realized with a lumped element structure. Let us assume that the formulae (7) and (8) are used. One can write the impedances of coupled transmission lines as follows:

$$
\begin{aligned}
& Z_{0 \text { o }}=\frac{Z}{1-k}, \\
& Z_{0 \mathrm{e}}=\frac{Z}{1+k} .
\end{aligned}
$$

The impedance $Z_{0 \mathrm{o}}$ is the square root of the ratio between the odd mode inductance and odd mode capacitance. To comply with Eq. (11) the capacitance and inductance of the odd mode should be written in a following way: 


$$
\begin{aligned}
C_{0 \text { o }} & =C(1-k), \\
L_{0 \text { o }} & =\frac{L}{1-k} .
\end{aligned}
$$

In a similar manner one can write formulae for the even mode capacitance and inductance

$$
\begin{aligned}
& C_{0 \mathrm{e}}=C(1+k), \\
& L_{0 \mathrm{e}}=\frac{L}{1+k} .
\end{aligned}
$$

From Eqs. (13)-(16) it is clear that line capacitances are coupled through admittance inverter with negative series capacitance, and line inductances are coupled through admittance inverter with series inductance [15]. Additionally, the velocities of propagation of the odd and even mode are the same and equal to the velocity of propagation of the single uncoupled line. The main drawback of the Oliver model is removed [17]. Now it is possible to draw the lumped element circuit of the unit section of coupled transmission lines and check the properties of the new model.

The model in Fig. 5a has negative capacitance $C_{\mathrm{s}}$. It is possible to simulate such a structure but it cannot be realized in practice. But at the single frequency one can replace the negative capacitance with positive inductance as shown in Fig. 5b. The inductances $L_{\mathrm{c}}$ are related to the negative capacitances $C_{\mathrm{s}}$ at the center frequency of the coupler. Moreover two inductances in parallel result in a single one - see Fig. 5c. With such a replacement one can obtain the lumped element circuit of the coupled transmission lines with all realizable elements but valid in a narrow band around the center frequency.

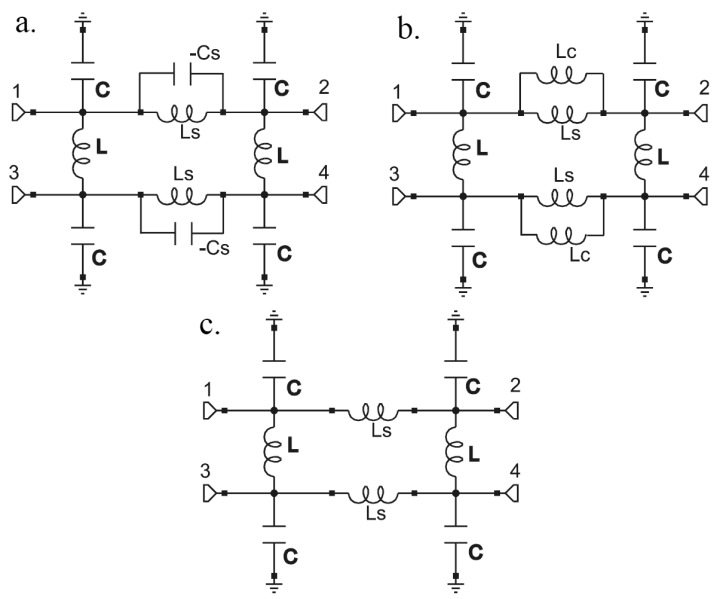

Fig. 5. New lumped element model of the unit section of coupled transmission lines in a directional coupler configuration. (b) Transformed structure of the new lumped element coupler. (c) Final circuit of new lumped element coupler.

Computed characteristics of the $6 \mathrm{~dB}$ new directional coupler with center frequency of $4.8 \mathrm{GHz}$ are shown in Fig. 6. Results are even better than initial characteristics

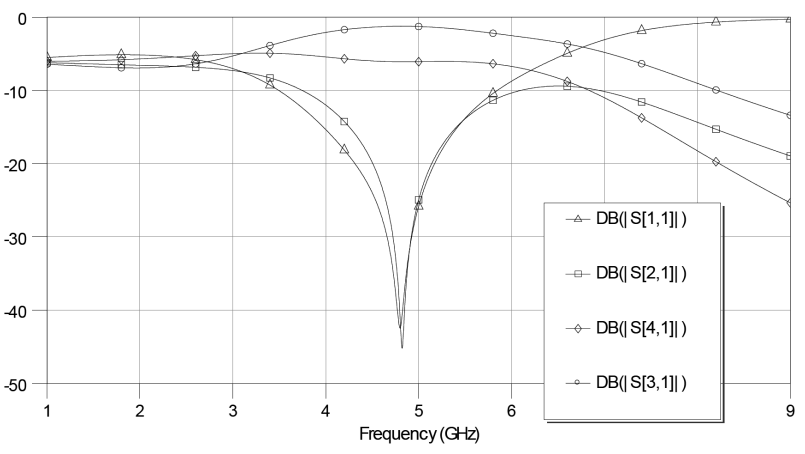

Fig. 6. Frequency characteristics of new lumped element $6 \mathrm{~dB}$ coupler operating at $4.8 \mathrm{GHz}$. Coupler elements: $L=1.45 \mathrm{nH}, C=1.122 \mathrm{pF}, L_{\mathrm{s}}=2.96 \mathrm{nH}$.

for the model with negative capacitance. In fact these characteristics are superior over other known lumped element couplers. The well-known lumped element coupler [18] in which coupling is provided through series capacitances is much worse taking into account flatness of $s 31$ and $s 41$ parameters.

\section{Conclusions}

The universal curve describing coupling structures has been found. The universal curve describing both coupled resonators and coupled transmission lines can be used to create and verify circuit models of coupled structures. The dependence of the coupling coefficient on the ratio between resonant frequencies squared or ratio between modal impedances should comply with the formulae (3) and (5). Taking the advantage of the existing models of coupled resonators the new model of coupled transmission lines has been developed. The new model has been used to create a new lumped element directional coupler. The characteristics of the new coupler are better than the characteristics of the traditional lumped element coupler. The universal curve can be also used to improve the accuracy of computed resonant frequencies and impedances. The same approach can be applied to unsymmetrical coupled resonators and transmission lines and can be useful in cross-talk analysis.

\section{References}

[1] S.B. Cohn, Proc. IRE 45, (Feb. 1957).

[2] G.L. Matthaei, L. Young, E.M.T. Jones, Microwave Filters Impedance Matching Networks and Coupling Structures, McGraw-Hill, New York 1964.

[3] R.J. Cameron, IEEE Trans. Microwave Theory Tech. MTT-47, (April 1999), p. 433.

[4] W. Gwarek, M. Celuch-Marcysiak, J. Phys. (France) III, vol.3., No. 3 (March 1993), p. 581.

[5] P.P. Silvester, R.L. Ferrari, Finite Elements for Electrical Engineers, 2nd ed., Cambridge Univ. Press, Cambridge 1990. 
[6] T. Ciamulski, W.K. Gwarek, IEEE Microwave Wireless Comp. Lett. 14, 298 (2004).

[7] G.W.O. Howe, Wireless Engineer, 9, 485 (Sept. 1932).

[8] K.R. Sturley, Radio Receiver Design, Part I, Chapman and Hall, London 1945, Ch. 4.

[9] Sz. Maj, A. Abramowicz, in: Proc. VIII KK TOiUE (8th Nat. Conf. Circuit Theory and Electronic Circuits), Wydawnictwo Politechniki Poznańskiej, Poznań 1985, p. 433 (in Polish).

[10] A. Abramowicz, in: Proc. XVIII Int. Conf. on Microwaves, Radar and Wireless Communications, MIKON 2010, Ed. B. Levitas, Geozondas, Vilnius 2010, p. 760.

[11] QuickWave, www.qwed.com .

[12] E.M.T. Jones, J.T. Bolljahn, IRE Trans. Microwave Theory and Tech. MTT-4, (Apr. 1956), p. 75.
[13] B.M. Oliver, Proc. IRE 42, (Nov. 1954), p. 1686.

[14] E. Costamagna, A. Fanni, M. Usai, IEEE Trans. MTT 41, No. 1 (Jan. 1993), p. 156.

[15] A. Abramowicz, Microwave Filters in Radiocommunication Systems, OWPW, Warsaw 2008 (in Polish).

[16] A.R. Djordjevic, M.B. Bazdar, T.K. Sarkar, R.F. Harrington, LINPAR, Artech House Inc., 1995 A.R. Djordjevic, M.B. Bazdar, T.K. Sarkar, R.F. Harrington, LINPAR for Windows: Matrix Parameters for Multiconductor Transmission Lines, Artech House, Norwood, MA 1995.

[17] A. Abramowicz, IEEE Trans. MTT 43, 1389 (June 1995)1389.

[18] P. Vizmuller, RF Design Guide, Systems, Circuits and Equations, Artech House, Boston 1995. 